\section{Design and Operation of a Charged Particle Beamline for the NOvA Test Beam Experiment}

David Duenas Tonguino ${ }^{1}$, Mike Wallbank ${ }^{1}$, Teresa Lackey ${ }^{2}$, Alexandre Sousa ${ }^{1}$, Andrew Sutton ${ }^{3}$, for the NOvA Collaboration ${ }^{1}$ University of Cincinnati, ${ }^{2}$ Indiana University, ${ }^{3}$ University of Virginia FERMILAB-POSTER-20-051-V le' CINCINNATI

Џ INDIANA UNIVERSITY

血 UNIVERSITY

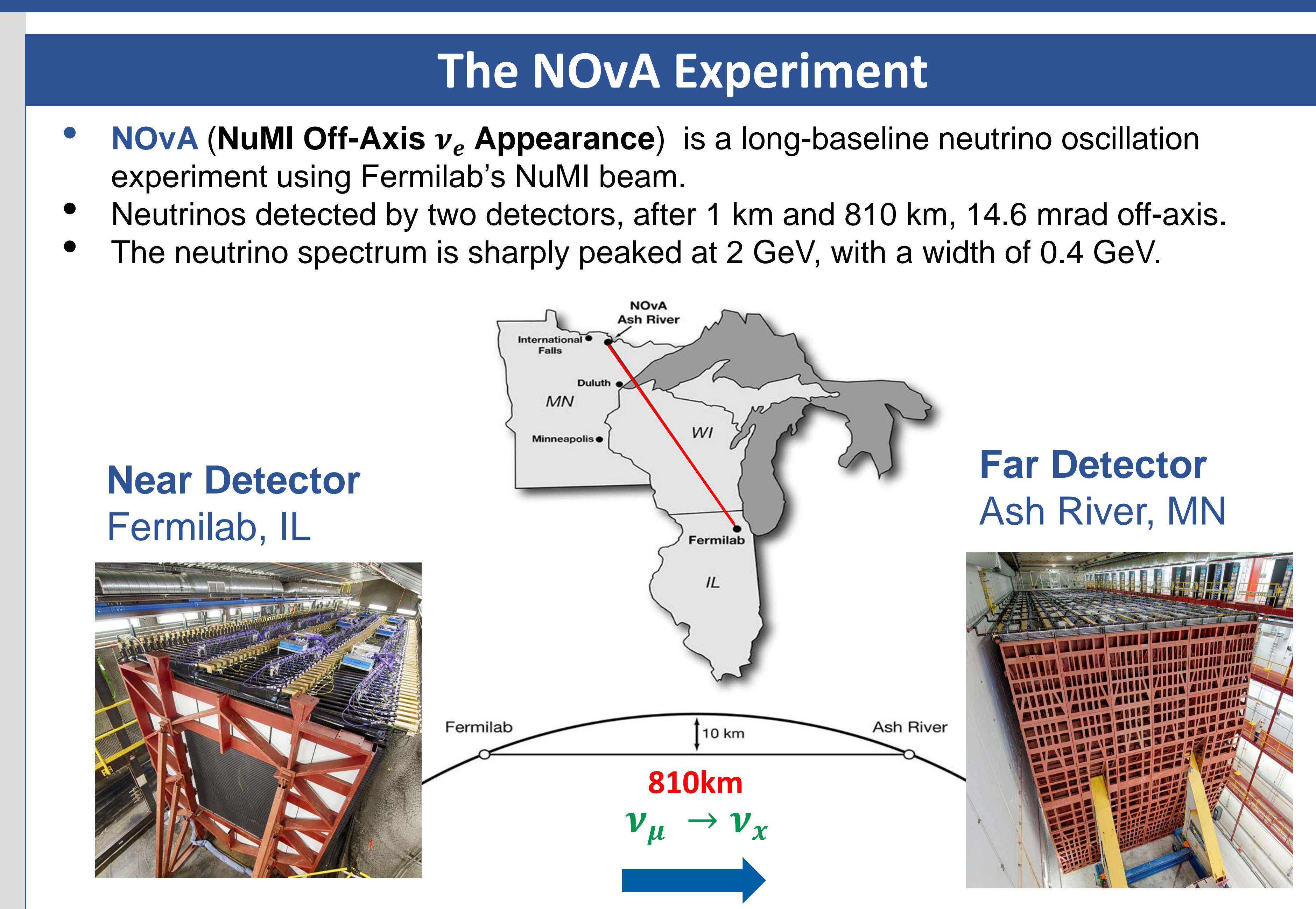

- NOvA is making precision measurements of the parameters governing neutrino oscillations, searching for evidence of $\mathrm{CP}$ violation in the lepton sector and aiming to resolve the mass hierarchy.

detector response, simulation and callown NOvA detector to improve understanding of uncertainties and improve event reconstruction and analysis.
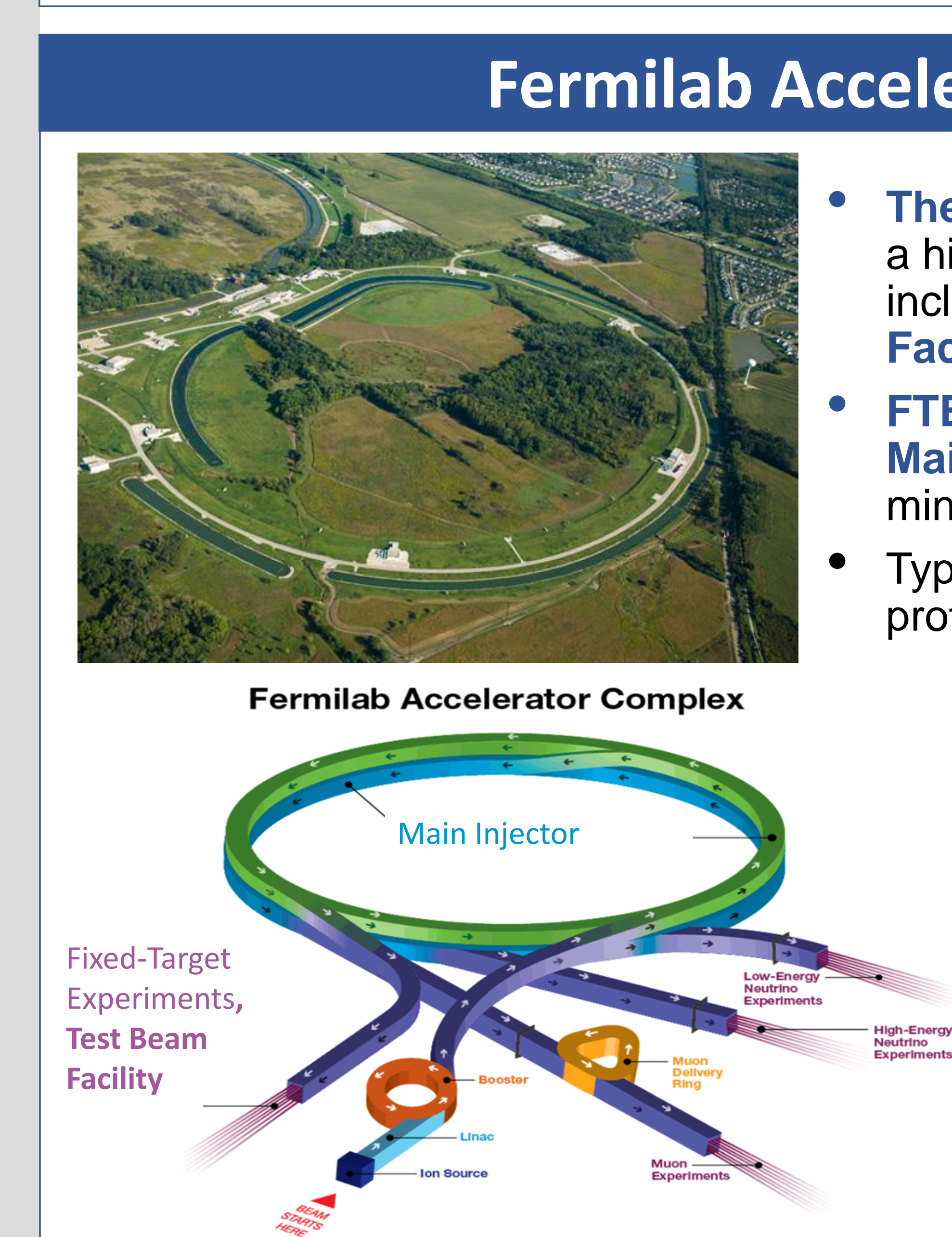

protons each spil

a high including NOvA and the Fermilab Test Beam Facility (FTBF).

FTBF receives $120 \mathrm{GeV} / \mathrm{c}$ protons from the Main Injector for a $4.2 \mathrm{~s}$ continuous spill every minute.

政 $1 \times 10^{9}$ and $1 \times 10^{10}$ FTBF

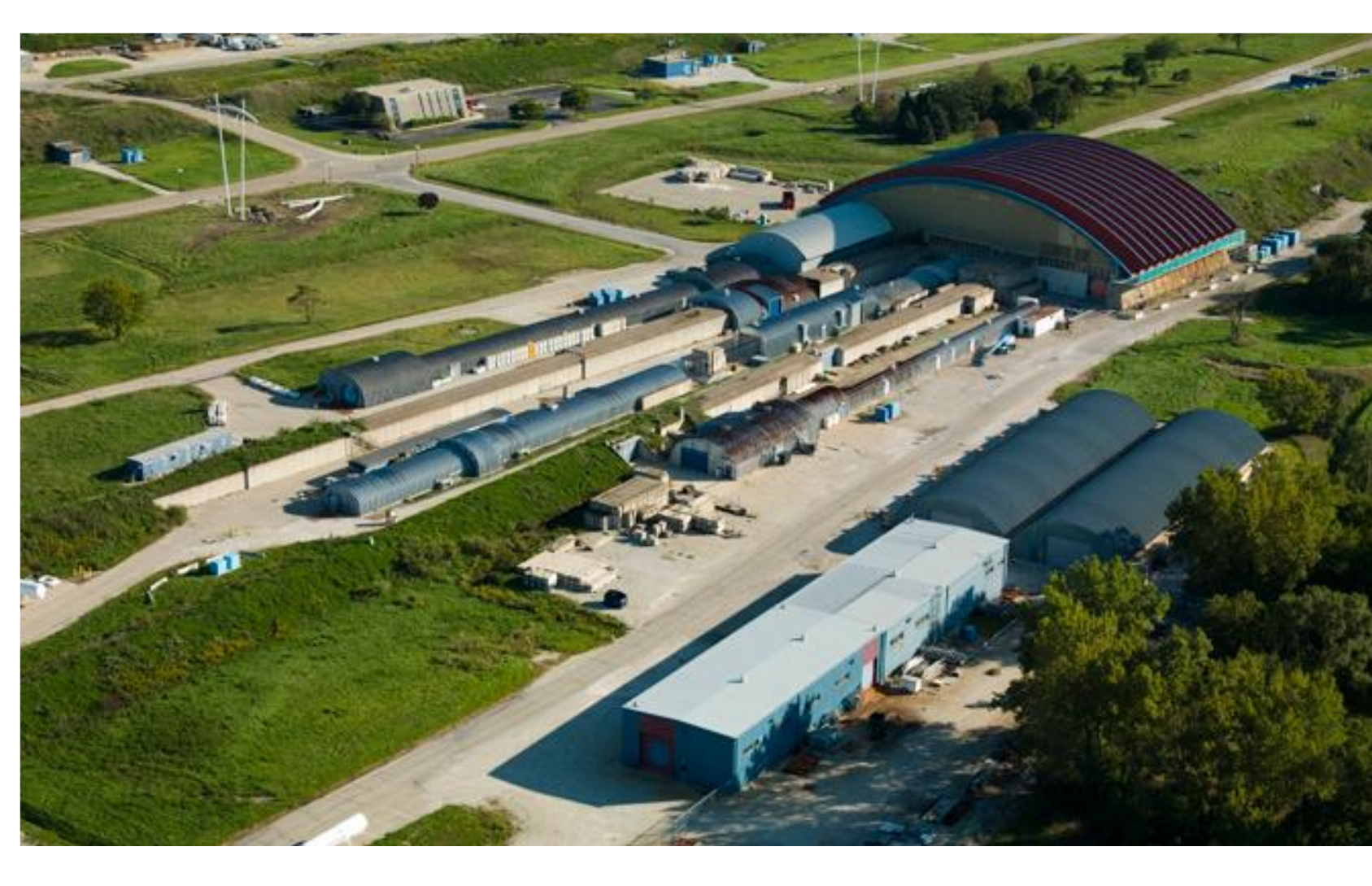

- The primary proton beam interacts with a primary cooper target in the facility creating - a secondary beam. - A tertiary beam is then produced through interactions of the secondary beam
particles with a second copper target.

hitps://www.fnal.gov/pub/science/particle-accelerators/images/accel-complex-animation.git

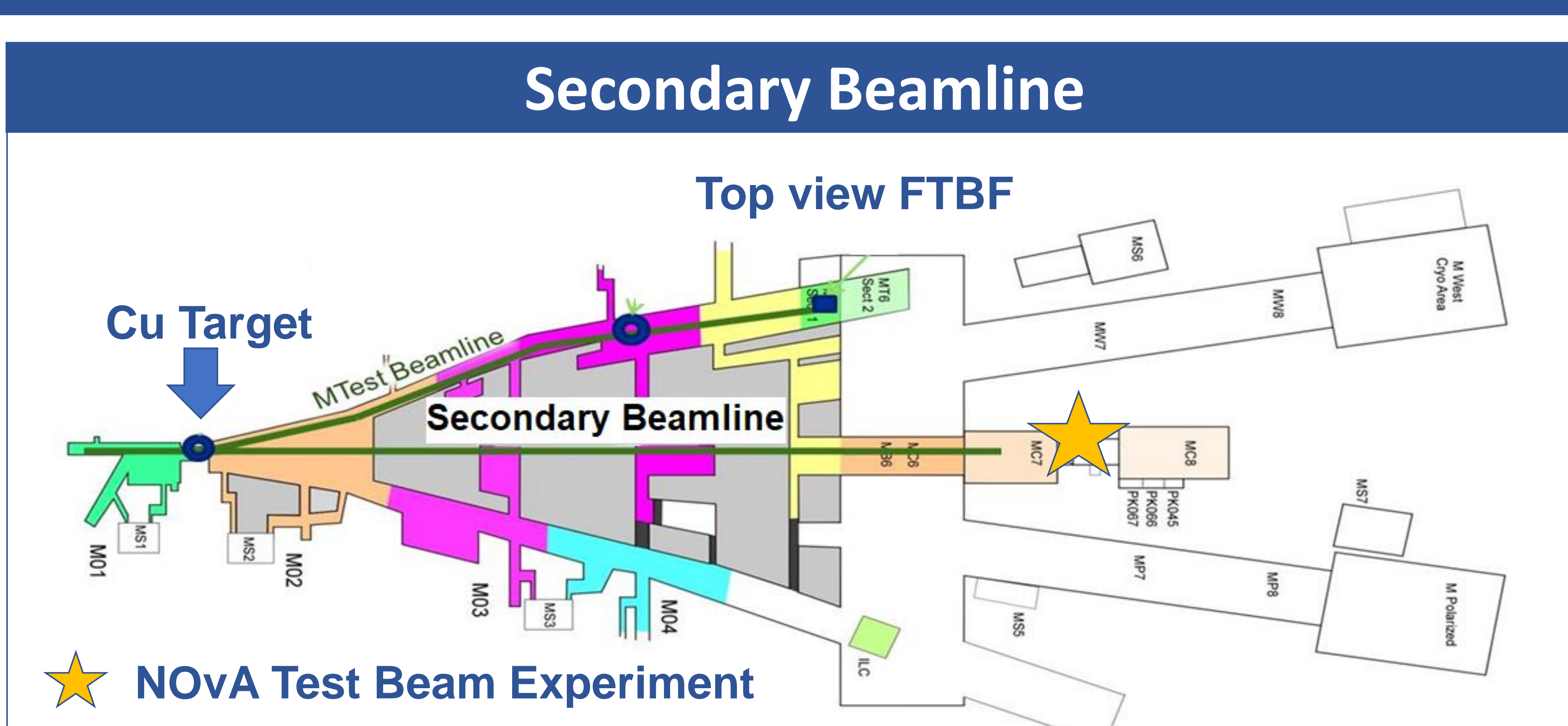

$120 \mathrm{GeV} / \mathrm{c} \quad$ Secondary Beamline, $64 \mathrm{GeV} / \mathrm{c}$ protons and pions $120 \mathrm{GeV} / \mathrm{c}$
protons
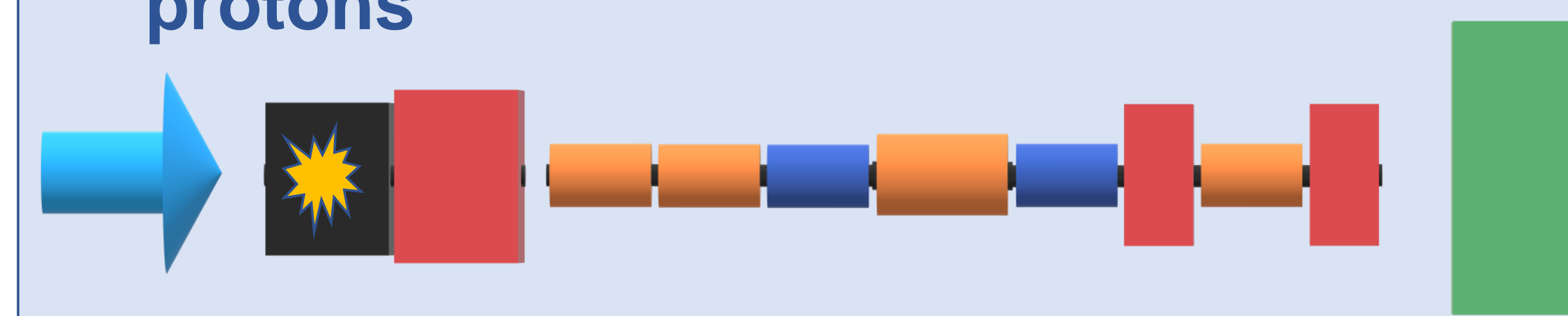

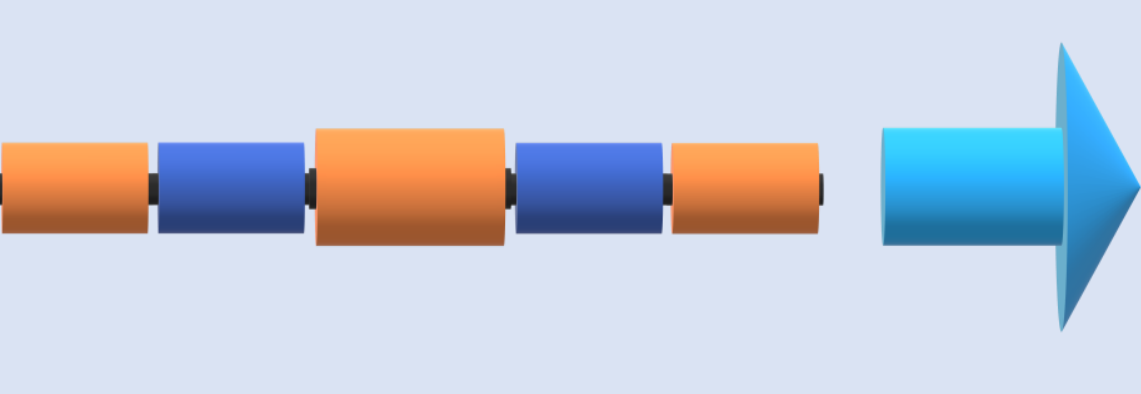

- Primary Cu Target: $120 \mathrm{GeV} / \mathrm{c}$ protons interact on the target to produce a - secondary beam of mainly protons and pions.

- Shielding Blocks and Collimators: Remove off-axis and off-momentum particles. Focus charged particles along the secondary beamline. Dipole Magnets: Used to select the momentum from 1 to $80 \mathrm{GeV} / \mathrm{c}$ by deflecting the particles. The Nova Test Beam Experiment has chosen $64 \mathrm{GeV} / \mathrm{c}$ to give the optimal tertiary production correct momentum and control the momentum bite of the beamline.

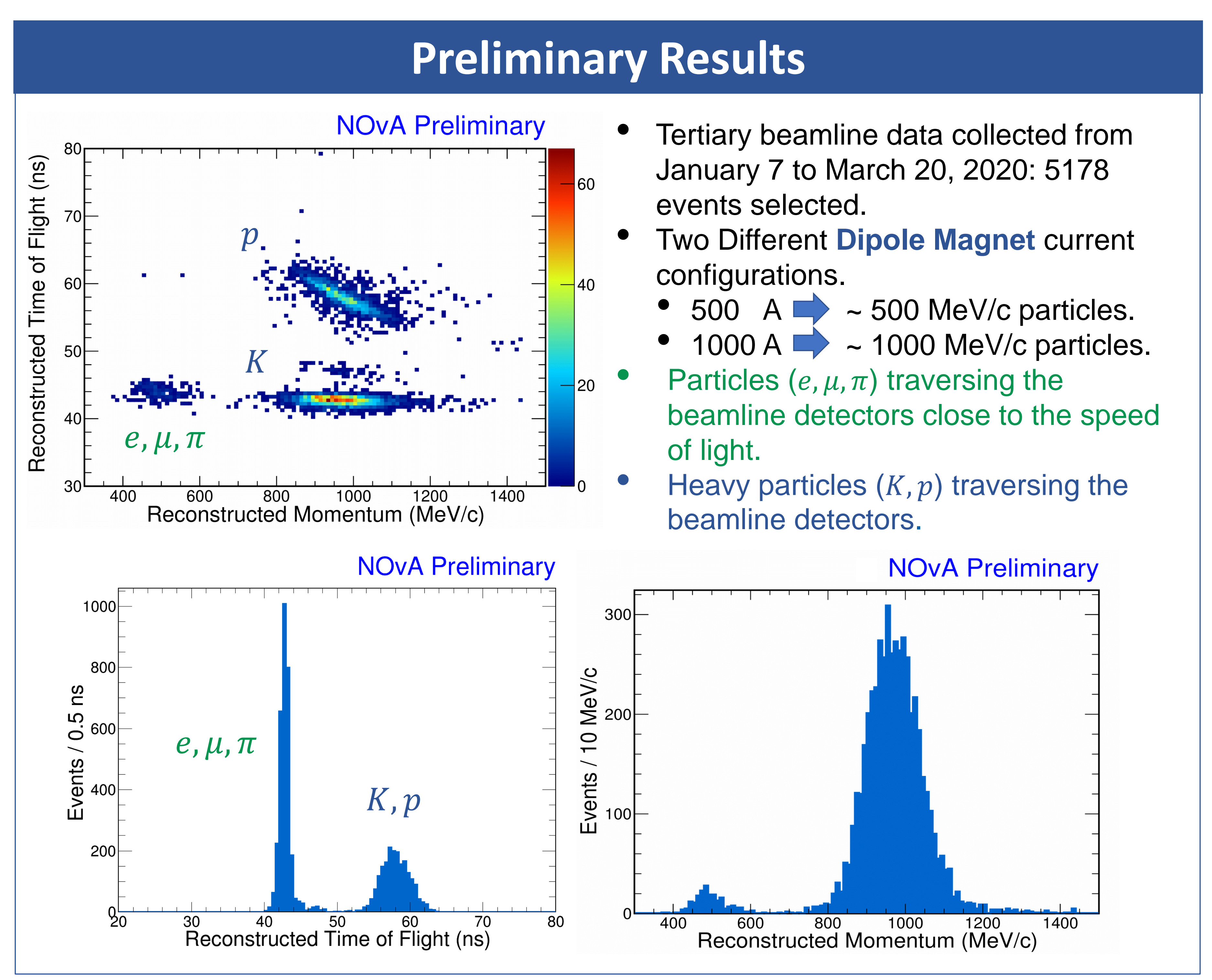

Tertiary Beamline

- The NOvA Test Beam Experiment analyzes particles with properties of interest to understanding the neutrino interactions in NOVA, charged particles with energies $0.2-2$

- In order to produce these particles, a tertiary beam is created from the $64 \mathrm{GeV} / \mathrm{c}$

- The tertiary beamline inclides

A des multiple detectors to characterize the particles before interaction in the NOvA detector and provide an external trigger for the readout

$\begin{array}{cc}64 \mathrm{GeV} / \mathrm{c} & \text { Tertiary Beamline, } 0.2-2 \mathrm{GeV} / \mathrm{c} \text { protons, } \\ \text { protons/pions } & \text { pions, kaons, muons, electrons }\end{array}$

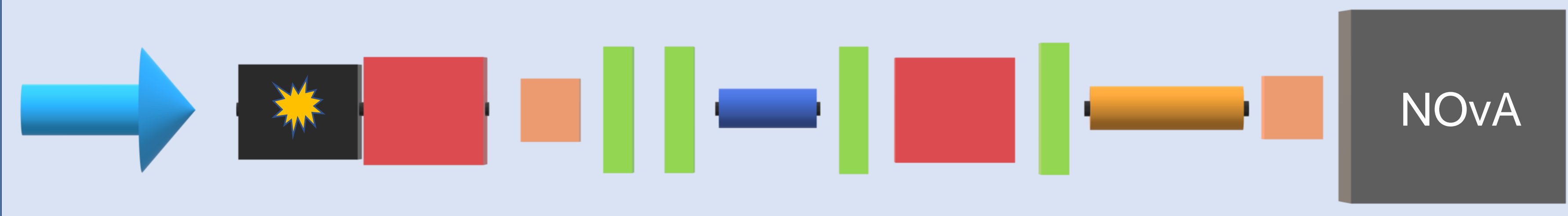

- Beamline components tag and select protons, pions, kaons, muons and electrons - in the momentum range of 0.2 to $2 \mathrm{GeV} / \mathrm{c}$

Secondary Cu Target : The secondary beam particles interact on the target to produce lower energy particles.

Time of Flight (TOF): Two scintillator paddles located at the upstream (US) and downstream (DS) ends of beamline to provide precise timing information and assist in

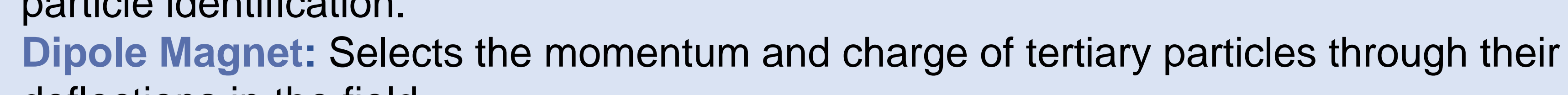
deflections in the field.

beamline.

Provide precise tracking of the particles through the

Cherenkov Detector: Tags electrons in the beam using Cherenkov light. The NOvA Test

-

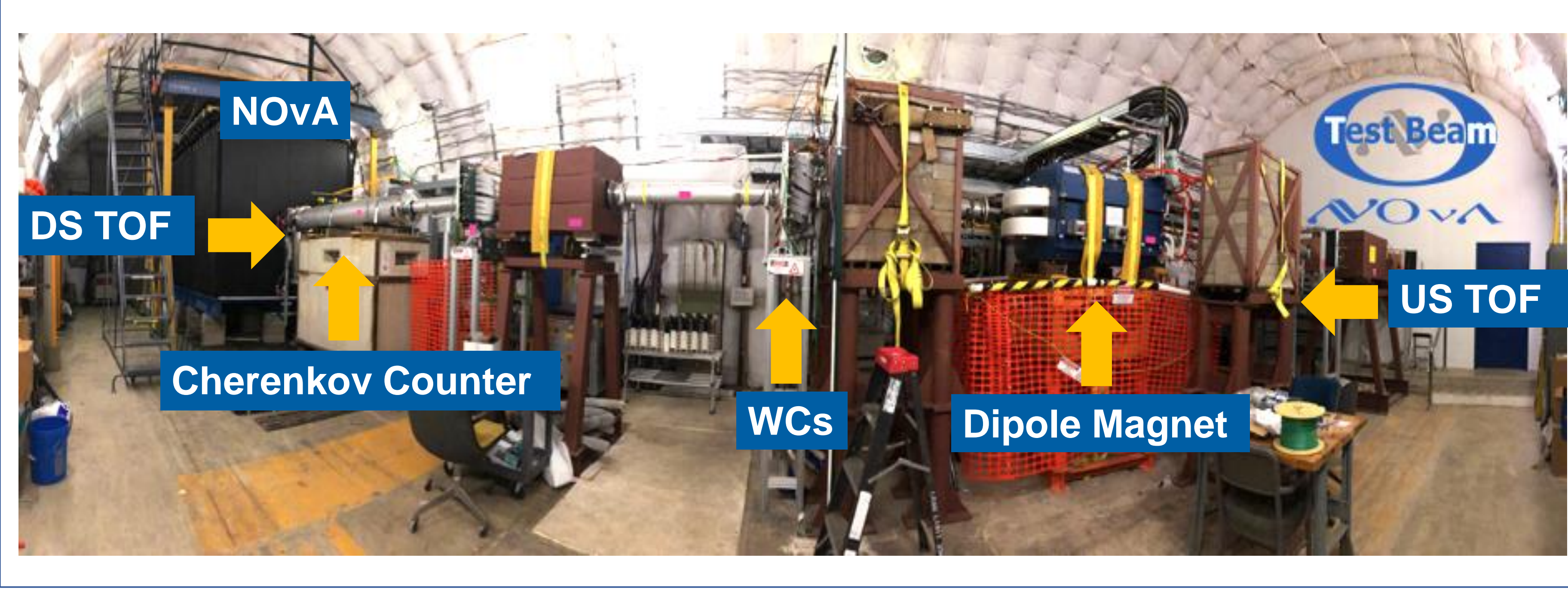

\section{Conclusions}

The NOvA Test Beam Experiment has in place a fully instrumented tertiary

Data taking operations from December 6, 2019 to March 20, 2020. Currently

characterizing charged particles.

Beginning to understand and analyze the data with a view to incorporating improvements into NOvA calibration, reconstruction and analyses. 